\section{GEOPHYSICS}

\section{European Colloquium}

from a Correspondent

ONE of the aims of the First European Earth and Planetary Physics Colloquium held at the University of Reading from March 29 to April 2 was to attract research students and others who find it difficult to obtain funds for long distance travel to attend scientific meetings. As it turned out, more than a third of the four hundred or so participants at Reading were younger scientists. The meeting was also successful in attracting Earth scientists from all over Europe; all Western European countries except Finland and Luxembourg were represented, and large groups attended from West Germany (46), France (31), Italy (27) and Holland (16).

The scientific programme was divided into six interdisciplinary symposia with invited speakers and three "open" sessions for submitted contributions. The importance of an interdisciplinary approach to many of the major problems of geophysics was clearly demonstrated. It was shown, for example, how studies of the dynamics of rotating fluids are applied to motions both of the Earth's liquid core and of planetary atmospheres; how physical chemistry and chemical petrology can contribute fundamental data on the composition of planetary atmospheres; and how petrological and geophysical approaches play complementary parts in the study of the physical properties of the Earth's mantle. Participants migrated freely among parallel sessions. Three successful "frontier" lectures were given in the evenings outside the normal programme, on topics in which major advances are being made.

Future policy was discussed at two open business meetings. Some senior scientists expressed reservations about the desirability of establishing a distinct European organization for geophysics, but most of the participants clearly felt this to be the simplest way in which regular European meetings, accessible to younger scientists, could be assured. Accordingly, it was formally resolved to establish an Association of European Geophysicists the primary purpose of which would be the organization, annually if possible, of the European Earth and Planetary Physics Colloquia. The programme of future meetings would broadly follow the pattern established at Reading, except that the "open" scientific sessions would be subdivided more along the lines found successful by the American Geophysical Union and the International Union of Geodesy and Geophysics, in order to ensure full participation by geochemists and others whose interests overlap conventional geophysics; it was felt that the rather idealistic subdivision of these sessions at the Reading meeting into planetary interiors, planetary atmospheres and interplanetary processes may have deterred many geologists and geochemists.

A broadly based steering committee was set up with the task of finding national representatives to facilitate communications, and to ensure that other organizations, including the European Association of Exploration Geophysicists, were kept fully informed of the plans of the new association. Representatives of the American Geophysical Union and the Royal Astronomical Society undertook to distribute information through the newsletters of their organizations.

As a regional geophysical meeting the Reading colloquium was an undoubted success. The proposals for future meetings of its kind deserved support of everybody with an interest in the dissemination of ideas among geophysicists and a concern for the training of European Earth scientists.
BRAIN FUNCTION

\section{Chemistry of Leurning}

from a Correspondent

TEN years ago, any meeting with behaviour in its title would have been unlikely to have a biochemist among its participants. But at a recent symposium on macromolecules and behaviour at the University of Birmingham on March 18 and 19, at least 50 per cent of the contributors were biochemists-a real indication that the biochemist is becoming more involved in the psychologist's problems, especially in the field of learning and memory.

The first session was concerned with neurochemical correlates of behaviour with particular reference to learning. Opening the session, Professor $H$ Hydén (University of Gothenburg) described some sophisticated techniques for measuring the uptake of labelled amino-acids into individual neurones and glia from discrete parts of brain. Rats were taught to use their unpreferred paw, during which training

\title{
Hamster Sarcoma Virus Identified
}

IN next Wednesday's Nature New Biology, Nowinski, Old, O'Donnell and Kingsley Sanders provide evidence that hamsters have their own antigenically distinct group of sarcoma and leukaemia viruses: chickens, mice, cats and some other species are already known to have their own private RNA tumour viruses (oncornaviruses). The hamster virus (HaSV) studied by Nowinski et al. is released into the medium by a tissue cultured line of hamster sarcoma cells established by Bassin et al. (Intern. J. Cancer, 3, 265; 1968). The sarcoma from which the cells were derived appeared in a newborn hamster following the injection of mouse cells infected with a murine sarcoma virus. The hamster sarcoma virus, however, does not infect mouse cells but, as has been shown by Zavada and Macpherson (Nature 225, 24; 1970 ), is very efficient in transforming the BHK21 hamster cell line, in vitro. The origin of the HaSV is therefore not clear; it may be a modification of the murine sarcoma virus or it may be a hamster virus stimulated by the inoculation of the infected mouse cells.

Nowinski et al. prepared rat antiserum against one of the major internal structural proteins (the group specific or gs antigen) of $\mathrm{HaSV}$ and showed that it reacted in immunodiffusion tests with hamster oncornavirus gs antigens, but not with comparable antigens derived from murine, feline, and avian oncornaviruses. These gs antigens are of general biological interest because they are present in the tissues of some apparently normal inbred chicken and mouse embryos. Moreover, Payne and Chubb (J. Gen. Virol., 3, 379; 1968) have shown that the expression of the antigen in chicken embryos is regulated by a dominant gene. Huebner et al. (Proc. US Nat. Acad. Sci., 67, 366; 1970) have shown that an analogous situation occurs in mouse embryos. Normal adult tissues do not contain detectable amounts of the gs antigen found in their indigenous oncornaviruses and immunologically mature animals are incapable of producing antibodies against this antigen-a fact that suggests these animals have had previous experience of the antigen in embryonic life and may continue to receive antigenic stimuli during adult life.

It is difficult to reconcile all these facts and decide whether the gs antigen is coded by cell or viral genes or both. Are the antigens normal cell components that have a role in certain stages of embryogenesis and which in oncornavirus infections are produced and incorporated into the virus particle? Or are, as has been suggested by Huebner, the cell genes the DNA proviruses of that particular species' oncornaviruses? It seems very likely that the virus particles carry the genetic information for their own gs antigens, because some chicken sarcoma viruses transform rodent cells and, although these cells may not produce infectious virus, they usually contain chicken gs antigen. An alternative but less likely explanation of this result is that particular strains of oncornaviruses always elicit the same antigen even in the cells of different species. 
there was an initial stimulation in the uptake of ${ }^{3} \mathrm{H}$ leucine into the hippocampal region. Hydén described how, as training proceeds, this enhanced uptake shifts to the cerebral cortex, while labelling in the hippocampus returns to normal. His study of S13, $\mathrm{S} 14$ and $\mathrm{S} 100$ proteins has also shown that the $\$ 100$ protein which, before learning, is isolated as one band on electrophoresis, is recovered as two bands after a learning experience.

Dr D. A. Booth (University of Sussex) discussed the effects of cyclohexamide, an inhibitor of protein synthesis, on memory consolidation. $\mathrm{He}$ argued that it was still open to question whether the disruption of long term memory by cyclohexamide was the result of the blockading of the consolidation process or was the result of negative reinforcement arising from aversive characteristics of the drug. Dr J. T. Rick (University of Birmingham) pointed out that the majority of studies concerned with biochemical correlates of learning make use of aversive conditioning stimuli in order to obtain rapid overlearning on the part of the animal. Stimulation, such as electric shock, is known to have significant effects on brain chemistry independent of any learning process. $\mathrm{He}$ argued that studies based on the extinction rather than the acquisition of a learned response, when such stimulation is absent, might overcome this difficulty.

The simpler nervous systems of invertebrates have been well exploited in this area of research as exemplified by the work described by Professor G. A. Kerkut and Dr G. W. O. Oliver (University of Southampton). Using the common garden snail (Helix aspersa) and the cockroach (Periplaneta americana) these workers described how, during learning, acetylcholinesterase activity in the nervous system increases in the former where the transmitter acetylcholine is chiefly inhibitory and decreases in the latter where it is only excitatory. Professor Kerkut went on to argue that one explanation of these data is that changes in interneuronal activity that must accompany learning may be affected by conformational changes in the enzyme rather than by differential rates of its turnover. The state of the enzyme, in other words, would act like a switch increasing or decreasing synaptic security.

The second session was concerned with modifications of brain chemistry in relation to behaviour. Dr $J$. Dobbing (University of Manchester) reviewed some of his studies on the effects of various early environmental conditions on future brain development and the extent to which such effects depend on just when these conditions occur relative to the normal time scale of maturation for the species. Dr G. Ungar (Texas Medical Center, Houston) dealt with the possibility of "learning" as a transferable phenomenon. $\mathrm{He}$ described the amino-acid sequence of a polypeptide extracted from the brain of a trained animal which, after injection, specifically aided the learning of a naive animal. Professor P. Mandel (Centre de Neurochimie, Strasbourg) was critical of such possibilities, and took the idea to the logical conclusion as he perceived it; that is, because the synthesis of RNA is only possible where the complementary DNA is already established, every unit of acquired information must already have a receptor-site in the brain.

\section{REVERSE TRANSCRIPTASE}

\section{Transforms Initioted}

from our Cell Biology Correspondent

THE extent to which research groups are falling over each other in the headling rush to get into the reverse transcriptase scene is reaching ludicrous proportions. In the circumstances, repetitious research is inevitable, but repetitious publication is not. Isn't it time for some sort of moratorium? For example, it might be agreed that after a first token publication-the claim to a stake-further publications should await the collection of more than the odd datum. But, of course, there is

\section{Macromolecular Stereopathology}

AN article by Greer and Perutz in next Wednesday's New Biology may suggest to the imaginative mind the birth of a new branch of clinical sciencediagnosis by crystallography. The number of recognized human haemoglobin variants now runs well into three figures. Most are only structurally, and not functionally, distinguishable from the normal molecule, and do not give rise to clinical symptoms. Others do their possessors no good at all: the most famous, sickle-cell haemoglobin, has an anomalous tendency to aggregate in the deoxygenated state, and comes out of solution with disastrous consequences for the red cell. Some others are aberrant in their oxygen equilibria: one, $\mathrm{Hb}-\mathrm{H}$, is generated by an imbalance in the concentrations of $\alpha$ - and $\beta$-chains, and is a tetramer made up of four normal $\beta$-chains, which are incapable of generating haem-haem interactions, and the contingent sigmoidal oxygen uptake curves. In a further set of variants an amino-acid substitution in a position intimately related to the binding of the haem to protein leads to other types of functional failure, in some cases (the haemoglobin-M variants) the complete inability of the associated haem to bind oxygen.

The species that Greer and Perutz have now examined is haemoglobin Rainier, which is a $\beta$-chain mutant with its substitution at the important, Cterminal end, where there is strong sequence conservation between all vertebrate haemoglobins. The conversion of tyrosine-145 to cysteine brings with it a substantially decreased haem-haem affinity and Bohr effect, and an increase in oxygen affinity. The characteristic disorientation of the crystal lattice of the oxyhaemoglobin on deoxygenation was observed in haemoglobin Rainier crystals, which indicates that its abnormality does not arise from any inability of the quaternary structure to change from the oxy-pattern to the deoxy-pattern. A comparison of deoxygenated haemoglobin Rainier with the normal adult protein showed a clean difference Fourier map, except in the region of the $\beta$-chain C-termini. The missing tyrosine is apparent as a negative peak of electron density, and the cysteine sulphur shows up as a strong positive feature. Moreover, the normal cysteine93 , which normally resides in the Fhelix, on the other side from the haem-liganded histidine, is rotated about the $\alpha-\beta$ carbon bond, so as to move the sulphydryl towards the pocket that normally contains tyrosine145. The alignment of the normal with the abnormal cysteine side chains signifies the formation of a disulphide bond between them. This event causes a local conformational convulsion, in which the $\alpha$-carboxyl group and the imidazole of the terminal histidine-146 are displaced, so as to break the ionpairs in which they are normally involved, and the residue is drawn into the $\alpha$-helix, in which it is found in myoglobin. Perutz and his co-workers previously demonstrated that one of the broken ion pairs is responsible under normal conditions for the alkaline Bohr effect.

Neither can the normal conformational change on deoxygenation described by Perutz, which involves the displacement of the tyr-145 side chain from its pocket, take its course. Moreover, model building suggests that in haemoglobin Rainier the $\alpha$-carboxyl group can form an adventitious ion-pair with the $\alpha$-amino group of the partner $\beta$ chain to provide a further source of stabilization of the oxy-form. The disulphide bond also presumably stabilizes the $\beta$-chain conformation, which may be the cause of the anomalous stability of haemoglobin Rainier towards alkaline denaturation. A "molecular disease" then, specified beyond Pauling's most optimistic predictions of more than twenty years ago. 